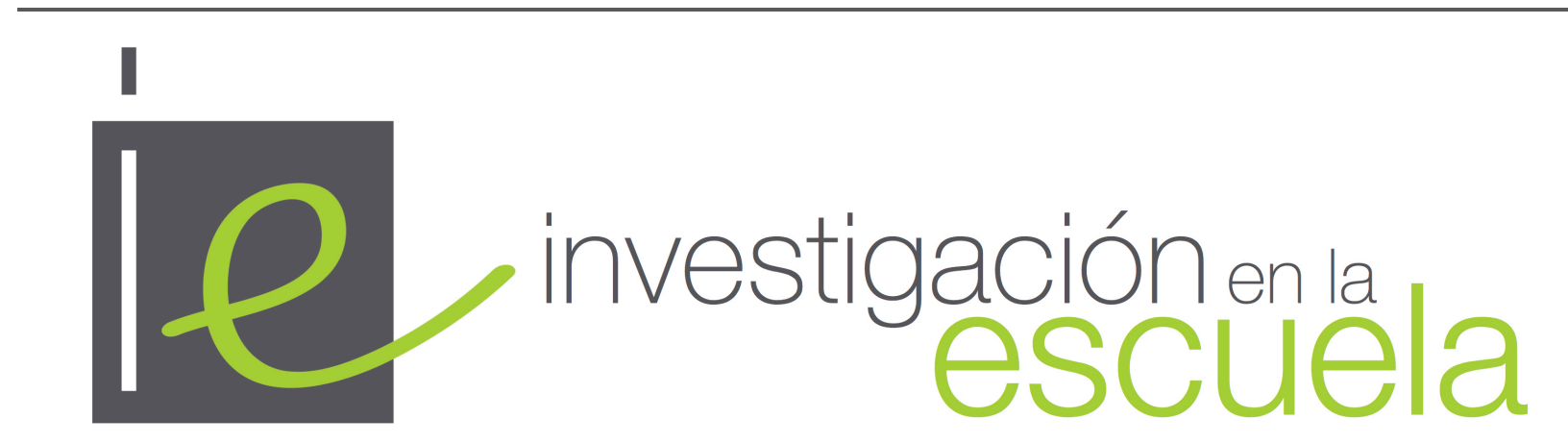

Revista internacional de investigación e innovación educativa

Número $100 \quad 30$ de abril de $2020 \quad$ ISSN 2443-9991

\title{
30 años de investigación sobre ciencia en el aula
}

\author{
Antonia Candela \\ Centro de Investigación y de Estudios Avanzados del IPN \\ México
}

Citación: Candela, A. (2020). 30 años de investigación sobre ciencia en el aula. Investigación en la Escuela, 100, 23-36. DOI: https://dx.doi.org/10.12795/IE.2020.1100.03

Resumen: En este artículo se presenta una revisión histórica con parte de mi trabajo de investigación etnográfica resaltando algunos aportes, en los que se abren temáticas teóricas que han resultado productivas para comprender los procesos de construcción social del conocimiento de ciencias en las aulas, sobre todo de educación básica en México. Estas temáticas incluyen a la argumentación, al razonamiento y a las formas de explicación que se desarrollan en la interacción discursiva entre alumnos y docentes. Se complementa este trabajo con los temas de construcción del consenso, así como el análisis del poder que los alumnos son capaces de desarrollar en la interacción discursiva escolar. Trabajos más recientes, producto de la profundización que permite complementar la mirada etnográfica con el análisis del discurso desde una perspectiva de Análisis Conversacional, destacan la participación de los alumnos como sujetos activos, construyendo una identidad como conocedores y competentes comunicadores en la construcción social del conocimiento en las aulas. Se describen los aportes de nuevos estudios como es la interacción entre pares en estudiantes universitarios de Física. Finalmente se plantea una reflexión sobre las actuales orientaciones educativas internacionales y su impacto negativo en la educación escolar.

Palabras clave: "Etnografía"; "ciencia en el aula"; "alumnos"; "interacción discursiva"; "argumentación”; “interacción entre pares". 


\section{0 years of research on science at the classroom}

Abstract: This article is a historical review of part of my ethnographic research, emphasizing some contributions and theoretical topics that turn out to be very productive in order to understand the social construction of science knowledge at the classrooms, specially at basic education in Mexico. Those topics include argumentation, reasoning and explaining that are developed among the discursive interaction of teachers and students. Consensus construction and the power students develop through the discursive interaction at the classroom complement this work. Within more recent studies the articulation of the ethnographic perspective with the analytical tools provide by a conversational analysis approach allowed me to show the students' as active participants developing an identity as knowledgeable and communicatively competent collaborators to social knowledge construction at the educational practices. Contributions of recent research topics as peer interaction in undergraduate students of physics are also described. A final reflection about actual international educational orientations and their negative impact on school education is state.

Key words: "Ethnography"; "science at the classroom"; "students"; "discursive interaction"; "argumentation"; "peer interaction".

\section{0 ânes de recherche dans la science en classe}

Resumè: Dans cet article, en m'aidant d'une partie de mon travail de recherche ethnographique, se sont présente une revue historique où je souligne quelques apports dans lesquels j’ai bien proposé des thématiques théoriques qui ont été enrichissantes pour comprendre les processus de construction sociale de la connaissance de sciences, surtout au sein de l'éducation élémentaire au Mexique. L'argumentation, le raisonnement et les formes d'explication qui se déroulent dans l'interaction discursif entre des enseignants et des élèves y sont compris. Dans ce travail, je complète ce travail avec les sujets de construction du consensus, même avec l'analyse du pouvoir que les élèves sont capables de développer lors de l'interaction discursive scolaire. Des travails plus récents, produits de l'approfondissement qui permet de compléter le regard ethnographique avec l'analyse du discours dans une perspective d'analyse conversationnelle mettent en évidence la participation des élèves. En tant que sujets actifs dans les salles de classe, ils créent une identité de connaisseurs et de communicateurs compétents dans la construction de la connaissance. J'esquisse de nouveaux sujets sur lesquels je travaille actuellement comme l'interaction en binôme chez des universitaires qui étudient la licence de Physique. Finalement, je réfléchis aux directives internationales actuelles en matière d'éducation et à leur impact négatif sur l'éducation scolaire.

Mots-clés: "Ethnographique"; "la science en classe"; "élèves"; "l’interaction discursive"; "argumentation"; "interaction entre pairs".

\section{Introducción}

La participación en la elaboración de los Libros de Texto de Ciencias Naturales para Niños y Maestros de Educación Primaria, material didáctico oficial de la Secretaría de Educación Pública de México de uso obligatorio para todas las escuelas de dicho nivel en los años 70 y 80, nos generó inquietudes sobre el uso de estos materiales en el contexto social de las aulas escolares, por la responsabilidad de tratar de transformar una práctica docente de la que no teníamos suficiente conocimiento. Esta inquietud se reforzó con la lectura de trabajos internacionales, como el de Yager y Penich (1983) en el que se mostraba, en una evaluación nacional de EU, que el constructivismo y el aprendizaje por descubrimiento (Bruner, 1963) de las reformas educativas de los años 60 y 70 tuvieron muy poca incidencia para cambiar lo que los niños sabían y las prácticas docentes como la 
catedra, el dictado de textos y la evaluación basada en la repetición de contenidos. Entre otras, se hicieron críticas al empirismo de muchos de estos materiales y al mito del "método científico" por el riesgo que representaban de hacer trasferencias mecánicas del proceso de investigación científica a las aulas escolares (Cañal y Porlán, 1987).

En este artículo describiremos varios estudios etnográficos realizados en diversas aulas de Educación Primaria. En esos trabajos se analizó la forma como se construye socialmente la ciencia en las aulas escolares desde la lógica que estos procesos tienen para los profesores y sobre todo para los alumnos. Los estudiantes han sido el foco de toda esta investigación por el interés de comprender su papel como destinatarios finales de la educación. Además de los recursos del enfoque teórico metodológico de la etnografía, un hilo conductor de estas investigaciones es el análisis del discurso de la interacción entre docentes y alumnos por asumir analíticamente al lenguaje como el medio privilegiado a través del cual se co-construye el conocimiento de la ciencia en la escuela (Mercer, 2001). En un apartado final también analizamos los procesos de resolución de problemas de tarea que realizan estudiantes universitarios de Física organizados en grupos de estudio. Todos estos trabajos permiten documentar el papel activo de los alumnos en la construcción social del conocimiento de ciencias en las aulas.

\section{Enfoques teórico-metodológicos: estudios socioculturales, etnográficos y análisis del discurso}

Los desarrollos psicológicos de la época nos acercaron a las concepciones vygotskianas que consideran el aprendizaje como un proceso sociocultural y sociohistórico. Para Vygotsky (1984) la comprensión del mundo físico está fuertemente influida por categorizaciones sociales que se interiorizan del contexto social, cultural e histórico y que influyen sobre las interpretaciones del mundo físico y social, que realizan los sujetos.

Asumir una posición constructivista social del aprendizaje escolar de las ciencias naturales, desde ese punto de vista, implica conceptualizar el proceso social de co-construcción del conocimiento científico como un proceso sociocultural que necesita estudiarse en el contexto propio de la educación: el salón de clases.

Trabajos, cada vez con mayor consenso dentro de la investigación educativa (Cicourel, 1974), planteaban que el aprendizaje significativo depende del contexto interactivo en el que es producido y no solo de las ideas previas de los sujetos. La perspectiva etnográfica permite analizar las claves de la comunicación entre los actores del proceso educativo para tratar de comprenderlo en su complejidad, sin estructurarlo, evaluarlo o controlarlos previamente (Rockwell, 2009). En la etnografía se busca entender el significado que los procesos educativos tienen para los actores (docentes y alumnos), en la trama de sus relaciones contextualizadas.

Después de los primeros trabajos etnográficos el interés por comprender a profundidad el significado de los contenidos de ciencias co-construidos en la interacción entre docentes y alumnos, nos condujeron a estudiar la interacción discursiva con la lupa epistemológica que representa el análisis del discurso desde la psicología discursiva desarrollada por Derek Edwards y Jonathan Potter (1992), sustentada en el análisis conversacional (Atkinson y Heritage, 1984). En esos trabajos relacionamos los aportes del estudio microsocial del discurso en el aula con la información etnográfica sobre el contexto sociocultural más amplio en el que se mueven los participantes.

Desde el punto de vista metodológico estos estudios son el resultado del análisis detallado de registros de interacciones discursivas que se obtienen de trascripciones de videograbaciones de clases de ciencias. Estas trascripciones se complementan con notas etnográficas tomadas por el observador, que es el mismo investigador. Las videograbaciones se obtienen después de estancias 
prolongadas en el contexto del salón de clases, de manera que los alumnos y los docentes se habitúen a la presencia de una videocámara fija y del observador tomando notas. Se pretende que el comportamiento de los participantes no se modifique significativamente.

\section{Acercándonos al aula}

En los años 90 iniciamos la investigación etnográfica, pero siguiendo todavía de manera cercana la propuesta de los Libros de Texto de Ciencias Naturales (LTCN). En el primer acercamiento retomamos conceptos de la teoría de las trasposiciones didácticas desarrollada por Ives Chevallard (1984) con algunas adaptaciones a este estudio. Analizamos las trasposiciones didácticas que ocurren en el proceso por el que un objeto de conocimiento para ser trasmitido en la escuela (en este caso, la forma en que aparece un contenido en los libros de texto) se convierte en un objeto de enseñanza (la forma de presentación del conocimiento por el docente en el aula). Estudiamos el desarrollo de las actividades experimentales en las aulas de una escuela de nivel socioeconómico bajo de la Ciudad de México (Candela, 1997). El siguiente es un ejemplo de lo que ocurre con un experimento que en los LTCN se planteaba como problema abierto, cuando esta actividad entra al contexto de la interacción en el aula.

Secuencia 1. La Combustión:

En una clase de $5^{\circ}$ grado de una escuela marginal de la Ciudad de México, se realiza una actividad experimental sugerida por el LTCN en la lección "La Combustión”, en la que se plantea: "Pon dos pedazos iguales de algodón en dos vasos de vidrio, enciende un pedazo de algodón con un cerillo y tapa los vasos. Cuando se apague agrega dos cucharadas de agua de cal a cada vaso. ¿Qué sucedió? ¿Cuál se puso lechosa y cuál no? ¿Qué quiere decir eso?”

Después de realizar la actividad al frente del grupo, el docente recorre el salón de clase mostrando los dos recipientes:

Mo: y ahora sí, vamos a ver qué diferencia hay entre las dos aguas de cal, ¿sí? ESTO ES para que no digan ustedes que el algodón se despinto, que el algodón hizo que el agua de cal se pusiera lechosa sino que fue exclusivamente la combustión. De este lado

Aoj: $\quad$ NO SE VE

As: je je je

Mo: de este lado está casi transparente el agua de cal Javier ¿sí?

Aoj: no

Mo: $\quad$ y de este lado se ve mas blanco a pesar de que tiene pedazos de papel quemado y vamos a...

Ao: $\quad$ yo no veo maestro

[hay otros comentarios incomprensibles pero el maestro no deja de hablar]

Mo: pasar por sus lugares para que los vean ¿sí? vamos a poner aquí un poquito más de agua de cal ahí está, vamos a pasar por acá ¿ya vieron?, aquí se ve un poquito más blanca el agua que aquí, aquí se ve todavía 
Mo: véanlo los dos, ¿ya lo vieron los dos?

Ao: no

Mo: sí, mira aquí se ve más blanca y aquí se ve casi

transparente todavía, como la hicimos en un

principio, ¿sí Maricela? SIMÓN, GUARDEN

SILENCIO ¿SÍ? por favor, ¿ya viste?, de este

Lado se ve más blanca el agua más lechosa

que de este lado, de este lado está completamente

transparente como cuando comenzamos ¿ya vieron?

está mas clara ésta ¿iverdad?

Aa: no [muchos niños hablan pero en voz baja y no se entiende]

Mo: más transparente ¿ya vieron que de este lado?

[a continuación aparecen 22 turnos en los que el maestro sigue insistiendo en que vean la diferencia pero ya ningún alumno interviene].

En esta interacción discursiva se nota que, en su presentación, el docente cierra la pregunta sobre la diferencia entre los dos recipientes con agua de cal, que en el LTCN era una pregunta abierta (¿Qué sucedió?). Él tampoco pregunta a los alumnos sobre la causa del fenómeno. Desde el inicio aclara lo que ellos "tienen" que ver en los dos recipientes (que el agua de cal en uno de ellos se pone lechosa) e incluso descarta lo que serían falsas explicaciones (no es porque se despinte el algodón) añadiendo lo que sería la explicación correcta de ese fenómeno (es por la combustión).

Esto es, la presentación que hace el maestro de la actividad experimental en el espacio social del aula, modifica el problema de describir y explicar lo que ocurre con el agua de cal en los dos recipientes por una trasposición que lo convierte en una demostración de que el agua de cal se pone lechosa y que eso es por la combustión del algodón. Sin embargo, y a pesar de la claridad de las orientaciones iniciales del docente, y de su insistencia posterior para que "vean" los cambios en el agua de cal, los alumnos le repiten una y otra vez que no ven nada, rechazando, hasta con el silencio, la presión del maestro. Se puede decir que, con su participación, los alumnos realizan otra trasposición de la actividad ya que impiden que ésta se construya en la interacción como una demostración.

Este es un ejemplo de que las propuestas didácticas pueden ser totalmente transformadas al entrar al espacio de interacciones discursivas que son las aulas escolares. En esta situación también se puede apreciar que la observación empírica, como procedimiento de validación del conocimiento, no es objetiva. Lo que "podemos ver" y sobre todo la manera como lo interpretamos depende de nuestros puntos de vista previos y del contexto particular en el que se realiza la actividad. Como observadora en esa clase, para mi era notoria la diferencia de transparencia del agua entre los dos recipientes.

Para los estudios de Sociología del Conocimiento Científico (Latour, 1988), la ciencia es una manera de interpretar la realidad natural, diferente de la cotidiana y de otras interpretaciones culturales, construida a partir de supuestos, teorías, conocimientos, lenguajes y prácticas culturales compartidas en una comunidad (Candela, 2013). Por tanto, la ciencia también es una cultura.

\section{E1 razonamiento, las explicaciones y la argumentación}

El trabajo de Erickson (1982) en el que considera que los alumnos en las aulas participan siguiendo la estructura de la participación social (EPS) y/o la estructura de la tarea académica (ETA), ha sido un referente importante para analizar el razonamiento de los niños en las aulas de educación básica. El razonamiento que sigue la dinámica de la interacción establecida con el maestro (EPS) es 
el que ponen en juego para tratar de entender cómo y cuándo participar en la clase. Seguir esta estructura es una forma de adquirir normas de comportamiento social y de ubicarse frente a las reglas del juego que definen la educación escolar. Por otro lado, el razonamiento de los alumnos que se centra en la lógica que para ellos puede tener el contenido se refleja en la estructura de la tarea académica (ETA).

En un artículo publicado en Investigación en la Escuela en 1990 (Candela, 1990), se analiza la interacción entre los alumnos y una maestra, en una clase de $5^{\circ}$ grado de una escuela pública en la que se realiza un experimento por equipos. En la actividad experimental se compara la flotación de un huevo en un vaso con agua simple, en otro con un poco de sal en el agua y en un tercero con agua saturada de sal. Esta clase muestra una gran participación de los alumnos que siguen su propia lógica para tratar de entender y dar una explicación del fenómeno. Hacen hipótesis iniciales en torno a cambios en el huevo (de tamaño o de peso) como factor causal de la flotación, mientras, en paralelo, toman en cuenta la lógica de la interacción social con la maestra para distinguir la densidad del agua como un elemento explicativo.

Esta situación contrasta con la actividad sobre el agua de cal, en la que su razonamiento sobre el contenido de los dos recipientes entra en conflicto con la lógica de la interacción que trata de imponer el maestro. Se muestra entonces, que el significado que los alumnos construyen sobre el contenido determina en gran medida su capacidad de articular estas dos formas de razonamiento y de incorporar las intervenciones docentes.

Trabajos sobre la capacidad de argumentación de los alumnos cuando sus ideas entran en conflicto con lo planteado por los docentes, por sus pares o con las preguntas que el contenido les sugiere, abrieron una línea de investigación muy productiva, que ha sido desarrollada posteriormente por importantes investigadores (Jiménez-Aleixandre y Puig, 2012), sobre la importancia de la argumentación en la construcción de la ciencia en contextos educativos. Por argumentación se entiende la articulación de intervenciones, dentro de un discurso, con la intención de convencer a otros sobre un punto de vista (Billig, 1987). Se estudia la argumentación desde una perspectiva émica que depende del significado de las acciones para los actores educativos, más que desde una concepción ética lingüística que tiene que ver con la lógica formal. En un ejemplo, ya clásico dentro de mi trabajo (Candela, 1991), se muestra la argumentación que se desata entre alumnos, en una clase de $5^{\circ}$ grado sobre "La Gravedad". Después de analizar la dinámica de los planetas en el Sistema Solar, una niña pregunta: ¿quién sostiene a la Tierra? La devolución de la pregunta que hace el maestro promueve la participación de los niños que se enfrascan en un debate argumentando sobre diversos modelos explicativos (al girar se genera aire que sostiene a la Tierra, como que la Luna nos tiene amarrados, pero pesa más la Tierra y jalaría a la Luna, hay gravedad en todo el espacio y eso sostiene a la Tierra), en el que se muestra su lógica de razonamiento para explicar un fenómeno desde sus diversas experiencias y conocimientos.

Este estudio muestra que la argumentación está implicada en la tarea y surge de los conflictos entre las diversas versiones que, sobre el significado del conocimiento, construyen los participantes en el contexto interactivo. Estas versiones generalmente son impredecibles. Por eso, las propuestas didácticas que proponen enseñar a argumentar, o enseñar otras "competencias", pueden terminar en la repetición sin sentido del procedimiento, por carecer de la relación que los participantes establecen con el significado que el contenido tiene para ellos, en el contexto de la interacción. Si bien la argumentación es uno de los enfoques más desarrollados en la actualidad para propiciar el razonamiento de los alumnos en la enseñanza de ciencias, se ha encontrado que las formas de aprender no son universales, sino culturales y que, por ejemplo, los indígenas aprenden más por observación (Paradise y Rogoff, 2009) y por "escucha radical” (Tobin, 2009) que por confrontación y argumentación de puntos de vista, como ocurre en contextos más occidentalizados. 


\section{El Poder de los alumnos en el aula}

Desde los años 70 hay consenso en la investigación educativa de que de la estructura discursiva dominante en las aulas escolares es la de interrogación, respuesta y evaluación (IRE) (Sinclair y Coulthard, 1975) y que esa estructura representa el control de los maestros en la interacción discursiva del aula porque son ellos los que preguntan y evalúan lo que se dice (Cazden, 2001; Wells, 1993) mientras los alumnos solo siguen las pistas para intervenir como se les sugiere. En este trabajo analizo el contenido, dentro de la forma lingüística de la estructura IRE para identificar si esa estructura le otorga siempre el poder al docente en el aula.

Desde el trabajo pragmático de Julie Diamond (1996) se considera que en el discurso coexisten un poder institucional que se mantiene relativamente invariable (en el caso de la institución escolar, el que se otorga a los maestros para decidir el tema de estudio, para organizar las actividades académicas o para otorgar calificaciones), y un poder local que es negociado en la interacción y que requiere del consenso activo de los participantes para ejercerse.

$\mathrm{Al}$ analizar el contenido del discurso encuentro que las preguntas de la estructura IRE no son siempre de los docentes. Los alumnos también preguntan sobre contenido y no solo sobre procedimiento (Cazden, 2001) (¿qué pasaría si los planetas se pararan?; ¿por qué no nos caemos (se refiere a la Tierra en el espacio)?; Maestra, ¿por qué si echamos fierro (al agua) se hunde y si echamos madera no, aunque esté muy pesada?) y sus preguntas inciden en la dinámica de la interacción discursiva del aula.

En relación a las respuestas de los alumnos, la Secuencia 1 es un ejemplo de que los alumnos no siempre siguen las orientaciones docentes para responder lo que se les pregunta, sobre todo cuando el significado del contenido que se aborda no es claro para ellos o entra en contradicción con sus ideas.

Además, los alumnos con frecuencia evalúan a sus compañeros, pero también lo hacen con los docentes y exigen explicaciones cuando no están convencidos, como lo que ocurre en una clase de $5^{\circ}$ grado después de una discusión sobre si el fierro o el plomo es el más denso. En ese debate muchos niños defendían que el plomo era más "pesado" mientras el maestro trataba de conducirlos hacia el fierro como el más denso.

Secuencia 5:

Mo: $\quad$ bueno (0.2) pongamos el fierro (.) por mayoría de opiniones, luego vamos a hacer una lista

Ao: ¿cómo vamos a saber si ya está bien?

Mo: A ver (0.2) tú Rubén tú pasas (.) a poner la segunda?

La intervención de este niño cuestiona implícitamente la versión sobre el conocimiento que plantea el docente y también el procedimiento de decidir cuál es la respuesta correcta por mayoría de opiniones. Los alumnos, son agentes activos en la escuela, que casi siempre tienen una opinión evaluativa de lo que hacen y dicen sus pares y sus maestros, aunque no siempre la expliciten. Vemos por tanto que aún dentro de la estructura IRE los alumnos pueden asumir y negociar el poder local con el docente, para incidir sobre la dinámica del trabajo en el aula, al preguntar sobre el contenido, al desviar las orientaciones del maestro con sus respuestas e incluso evaluando a compañeros y al profesor. Aún con su silencio se pueden negar a seguir las indicaciones docentes y a aprender.

En síntesis, parte de lo mostrado en estos estudios sobre la interacción discursiva en clases de ciencias de la escuela primaria en México es:

1) Cuando las propuestas didácticas entran al espacio social de las interacciones entre los docentes y los alumnos en las aulas, se modifican de formas impredecibles por los múltiples factores humanos e institucionales que operan en la escuela. Por eso, las propuestas didácticas difícilmente pueden predecir las prácticas y el conocimiento que se puede construir en el contexto social. 
2) La memorización de datos, la guía, la demostración o la información previa sobre las conclusiones a las que "hay que llegar", no impiden el razonamiento divergente de los alumnos, sobre todo cuando su reflexión tiene un referente alternativo a la lógica que demanda la dinámica de la interacción con el maestro, como son las actividades experimentales, y se les otorga el tiempo necesario para su co-construcción reflexiva.

3) Los alumnos son sujetos activos en las aulas y participan co-construyendo el conocimiento sobre temas científicos, cuando el contenido les resulta significativo. En las aulas no siempre los profesores tienen el poder sobre el conocimiento que se construye socialmente ya que los estudiantes también contribuyen con versiones alternativas que negocian, argumentan, evalúan o cuestionan aun dentro de la estructura IRE. Si se analiza la estructura del discurso sin tomar en cuenta el contenido del mismo se puede llegar a conclusiones que no reflejan la influencia de los actores educativos en la construcción del conocimiento.

\section{Interacción entre pares en la resolución de problemas de Física a nivel universitario}

En los últimos años realizamos investigaciones etnográficas en clases de Física de la Universidad Nacional Autónoma de México (UNAM) (Candela, 2010). Uno de los referentes más importantes para estos estudios es el de Jan Nespor (1994) que amplia los estudios de la construcción del conocimiento y de las prácticas realizadas en interacciones cara-a-cara con las interacciones de los sujetos con otros tiempos y otros espacios constituyendo así redes por donde los alumnos tienen que aprender a moverse. A continuación describiremos la forma como resuelven problemas de tarea los estudiantes universitarios de física.

La resolución de problemas es una práctica central de la formación de los físicos, que en la carrera universitaria generalmente se realiza en grupos de estudio. A decir de varios estudiantes entrevistados, sin su participación en un grupo de estudio no hubieran podido terminar la carrera. La formación de grupos de estudio para resolver las tareas es una práctica que ocurre prácticamente en todos los países, por la necesidad de apoyarse con un trabajo colectivo frente a la complejidad del contenido disciplinario (Nespor, 1994). Analizar cómo interaccionan los estudiantes en estos grupos de pares tiene importancia para entender cómo aprenden.

La mayoría de los escasos trabajos que estudian la interacción entre pares a nivel universitario, se centran en actividades organizadas institucionalmente en las que se entrena a alumnos avanzados para asesorar el trabajo de sus compañeros, pero estos estudios aportan pocos resultados favorables en cuanto al aprendizaje de los estudiantes (Topping, 2005). En México son los propios alumnos los que forman los grupos de estudio sin injerencia institucional y estos grupos generalmente se mantienen a lo largo de toda la carrera. Analizo observaciones y grabaciones de estos grupos de estudio que resuelven tareas de un curso de termodinámica del 3er semestre (Candela, 2018).

\section{Articulación individual y colectiva como proceso social constructivo}

El trabajo de resolución de problemas de tarea en todos los grupos de estudio observados presenta una característica notoria en la forma como se vinculan las acciones individuales con las colectivas. El siguiente es un ejemplo:

Secuencia 7:

Tres alumnos se citan en la biblioteca para trabajar sobre la tarea semanal. Rolando les explica a Adela y a Adán la estrategia que él uso para resolver uno de los problemas que los otros dos no habían podido realizar individualmente. Después de la explicación 
ambos por separado checan sus notas de clase y realizan las operaciones matemáticas necesarias para resolver el problema. Finalmente, los tres comentan conjuntamente el resultado para verificarlo.

Resalta la diferencia en el trabajo realizado por cada alumno, pero también es notable la ausencia de competencia entre ellos y la colaboración para apoyar el proceso constructivo de sus pares, pero sin sustituirlo. Rolando aporta la estrategia para plantear la resolución a sus compañeros, pero ellos diseñan los recursos matemáticos y realizan las operaciones para finalmente comparar sus resultados. El trabajo individual y colectivo son complementarios y llevan implícito el apoyo y la mutua supervisión, pero no se elimina uno por otro.

\section{Rotación de roles}

Por otro lado, el papel que juegan los participantes en el grupo de estudio no es siempre el mismo, ni siquiera la presencia de todos los estudiantes en el trabajo del grupo es simultanea todo el tiempo.

Secuencia 8:

Ruth se acerca a Rolando que está trabajando en una mesa de la biblioteca y le pregunta por el problema 4. El le da una hoja con la solución del problema que le dejó Lola que es miembro del grupo aunque en ese momento no está porque tiene otra clase.

En este caso es Lola la que apoya el trabajo de Rolando, dejando incluso a su juicio la posibilidad de que éste lo comparta con otros miembros del grupo de estudio, mientras ella se va a otra actividad. Si bien Rolando parece más competente que sus compañeros en la secuencia 7, en esta secuencia Lola aparece como la más competente frente a un nuevo problema. Así los roles en términos de pericia parecen rotarse permanentemente, en algunos casos siendo las mujeres las que mejor resuelven un problema, o parte de él, y en otros son los hombres. También en esta situación se observa la colaboración entre los alumnos, así como la variación continua entre los miembros del grupo de estudio que trabaja simultáneamente, lo que les da flexibilidad a estos grupos para adaptarse a diversas condiciones de comprensión, de tiempo disponible para realizar las tareas y de colaboración con sus pares. De esta manera el grupo de estudio representa una forma de enfrentar la diversidad de condiciones de trabajo, cognitivas e incluso socioeconómicas (por ejemplo, algunos tienen necesidad de trabajar mientras hacen la carrera). Esto disminuye el fracaso de los estudiantes, abre oportunidades para los diferentes y permite mayor continuidad en la carrera.

\section{Haciendo la tarea como "bricoleurs"}

Otra característica del trabajo de los alumnos de física en sus grupos de estudio es la dinámica de las interacciones discursivas: sobre qué, cuándo y cómo se realizan. Erickson (2004) denomina ser un "bricoleur": alguien que adapta las acciones a la situación concreta para realizar un trabajo de manera efectiva y en el tiempo de oportunidad, para hacer lo correcto en lo que el tiempo correcto requiere y con las "herramientas culturales" (como los recursos matemáticos, en este caso) disponibles en el contexto. Esta definición es sugerente para analizar el siguiente ejemplo:

Secuencia 9:

Adela y Adán están trabajando individualmente y de vez en cuando alguno le hace preguntas breves a otro como: “ ¿Coseno de pi es cero verdad?" Cada vez que Adela pregunta algo, Adán se voltea para ver si lo que quiere ella es verificar un resultado. Él ve lo que ella hizo, lo compara con su propia tarea y le dice que a él le sale lo mismo pero sin un "npi". Adela revisa su tarea y le explica a su compañero de donde viene el "npi". Adán hace correcciones y le pide que lo vuelva a confirmar, Adela lo revisa y le dice que graficó las ecuaciones en Maple y que sí funciona, le explica como quedaron las gráficas. 
En este caso, como en otros se ve en detalle que los alumnos están siguiendo un proceso individual de resolución del problema, pero en cientos momentos requieren y solicitan información precisa (¿Coseno de pi es cero verdad?) para poder continuar con el trabajo que están realizando. En este sentido están actuando como "bricoleurs", o sea, adaptando su apoyo a las necesidades concretas en los momentos concretos. Además, la acción de verificar si tienen el mismo resultado, no los conduce a copiar el procedimiento del compañero sino a realizarlo de nuevo y en todo caso a defender una versión argumentando en base a recursos teóricos adicionales. Estos alumnos realizan un trabajo de mutua supervisión y de escucha crítica en la que no se acepta una sugerencia o información hasta que no se comprueba que es correcta, con recursos complementarios (como es la gráfica de las ecuaciones de Maple) para entender el procedimiento.

Estos grupos de estudio son una mediación entre la acción colectiva de la enseñanza en el aula y el aprendizaje individual, colaborando a la compresión de todos. Se puede observar que los alumnos o alumnas más competentes ayudan a otros a entender y resolver los problemas de tarea, pero estos roles se modifican permanentemente ayudando a evitar la jerarquización entre estudiantes. Por otro lado, el trabajo en el grupo de estudio para resolver la tarea es complementario y de mutua supervisión sin sustituir la actividad individual y la responsabilidad de cada alumno por la resolución de los problemas, pues las tareas entregadas generalmente tienen resultados diferentes. Aunque esta forma de trabajo no siempre ocurre, en los grupos de estudio para resolver las tareas domina la colaboración sobre la competencia. Las interacciones entre los estudiantes, siguiendo una práctica que se parece a la de un "bricoleur", ayuda a reducir las diferencias intelectuales, de condiciones de trabajo y de aptitudes de los alumnos porque satisface las necesidades individuales de comprensión y/o de información por la intervención de los pares en el momento de oportunidad, o sea, cuando y como se necesita.

\section{Reflexiones}

Durante estos años nuestro trabajo se ha centrado en tratar de comprender las condiciones del trabajo escolar y la dinámica de las interacciones sociales que mejor contribuyen a la construcción del conocimiento de ciencias en la escuela. Este propósito tiene el fin de recoger la voz de los participantes en ese complejo contexto que son las aulas (Candela, Rockwell y Coll, 2004), sobre todo, la lógica de las contribuciones de los alumnos a la co-construcción del conocimiento de ciencias. La apuesta ha sido por tratar de articular los avances de la investigación didáctica con las mejores prácticas recogidas desde la perspectiva de los actores en condiciones naturales, esto es, modificando lo menos posible su trabajo cotidiano (Candela, Naranjo y De la Riva, 2014). Tratar de llevar estas prácticas cotidianas a las propuestas educativas permite hacerlas más cercanas a las posibilidades de incorporarlas al quehacer docente ya que parten de contextos reales y son experiencias que, aunque no sean muy frecuentes, han mostrado su posibilidad de realizarse en la práctica.

Si bien, la agencia de los estudiantes en los procesos educativos ha sido el foco de esta investigación, la participación no podría desarrollarse si los docentes no la propiciaran y promovieran en las aulas. Trabajos etnográficos comparativos (Macias, 1992) muestran que en México el valor social de la educación tanto para los alumnos como para los profesores es notablemente mayor que en otros países. Esto conduce a un mayor compromiso de estos actores para mejorar las prácticas educativas, en la medida de sus posibilidades.

Una revisión de las prácticas educativas a lo largo de estos 30 años me permite observar que los docentes incorporan aquellas orientaciones didácticas de los proyectos educativos cuando les 
resultan útiles en la práctica, mientras mantienen otras prácticas a través de los años por las mismas razones, aunque éstas no sean recomendadas. Por ejemplo, actualmente se realizan más actividades experimentales en clases de ciencias que en los 90 y los maestros son más conscientes de la importancia de propiciar la participación de los alumnos en la construcción social del conocimiento escolar. Sin embargo, el enorme incremento de contenidos y de actividades de las propuestas oficiales actuales, la cantidad de demandas administrativas que los docentes tienen que realizar ahora y sobre todo la aplicación continua de diversas evaluaciones estandarizadas a alumnos y maestros, han disminuido el tiempo disponible para la enseñanza y la posibilidad de realizar un trabajo reflexivo. La disponibilidad de tiempo para poder enseñar se ha convertido en la demanda principal de los profesores que han sufrido un deterioro notorio en sus condiciones de trabajo.

Algunos de los factores que conducen a esta situación son principalmente de política educativa. Es significativo el impacto negativo que han tenido las políticas privatizadoras impulsadas internacionalmente por organizaciones como la OCDE, que abren la educación al mercado y ponen la ganancia por encima de las necesidades de formación para el desarrollo humano. Estas políticas que proponen la aplicación de evaluaciones estandarizadas a maestros y alumnos, con la pretensión de mejorar la "calidad" de la educación se definen como empresariales y fallidas por una de sus creadoras (Ravitck, 2010), que reconoce que tienen un impacto muy negativo en la educación. Entre sus efectos está el desplazar al currículo al enseñar para pasar la prueba, el pretender evaluar aprendizaje sin evaluar enseñanza, el incrementar la marginación de los sectores más desfavorecidos por imponer una evaluación igual (estandarizada) para los desiguales. Además, las pruebas no analizan los procesos educativos y sus resultados descontextualizados no aportan elementos para comprenderlos y tomar medidas para corregirlos. Finalmente, estas políticas responsabilizan a los profesores de los problemas educativos afectando su imagen pública y su autoestima. Los factores emocionales como la seguridad en sus propias acciones y conocimientos y la autoestima están teniendo una incidencia muy negativa en la docencia y sería conveniente estudiarlos con mayor detenimiento. Todo esto dificulta que los profesores asuman la flexibilidad y el tiempo de trabajo que requiere la vinculación de los contenidos con las necesidades de los alumnos en los diferentes contextos socioculturales y los procesos de construcción social del conocimiento (Candela, 2018).

Lamentablemente estas políticas han contribuido a un deterioro significativo de la educación, a pesar de la lucha en su contra que los maestros de educación básica de México hay desarrollado ya por varios años.

\section{Referencias}

Atkinson J.M. \& Heritage, J. (1984). Structures of Social Action: Studies on Conversation Analysis. Cambridge: Cambridge University Press.

Billig, M. (1987). Arguing and Thinking: A Rethorical Approach to Social Psychology. Cambridge: Cambridge University Press.

Bruner, J. (1963). El proceso de la Educación. México: UTEHA.

Candela, A. (1990). Investigación etnográfica en el aula: El razonamiento de los alumnos en una clase de Ciencias Naturales en la escuela primaria. Investigación en la Escuela, 11,13-23.

Candela, A. (1991). Argumentación y conocimiento científico escolar. Infancia y Aprendizaje, 55, 13-28.

Candela, A. (1997). Demonstrations and problem-solving exercises in school science: Their transformation within the Mexican elementary school classroom. Science Education, 81, 497-513.

Candela, A. (2010). Time and Space: Undergraduate Mexican Physics in Motion. Cultural Studies of Science Education 5, 701-727. Disponible en: 
http://www.springerlink.com/content/t703003671077480/?p=f0c7abdb0ab64f30acd445bd $\underline{543 f 3548 \& \mathrm{pi}=10}$

Candela, A. (2013). Dialogue between cultures in 'Tzeltal teachers' cultural discourse: co-construction on an intercultural proposal for science education. Journal of Multcultural Discourses. http://dx.doi.org/10.1080/17447143.2012.756492

Candela, A. (2018). Peer organized study groups: Successful learning interactions in Mexican undergraduate physics. Learning, Culture and Social Interaction, 11-21 https://doi.org/10.1016/j.lcsi.2018.03.007

Candela, A. (2018). Impacto de la fallida reforma educativa en el Sistema de educación básica de México: Alternativas. En C. Navarro (Coord.), Reforma sin Futuro y Resistencia magisterialy popular (pp. 323-355). Horizontes Educativos: Universidad Pedagógica Nacional.

Candela, A., Naranjo, G. y De la Riva, M. (2014). ¿Qué crees que va a pasar? Las actividades experimentales en clases de Ciencias. México: Ediciones SM-Cinvestav. Colección Somos maestros, Serie Enseñar y Aprender.

Candela, A., Rockwell, E. \& Coll, C. (2004). What in the world happens in classrooms? Qualitative Classroom Research. European Educational Research Journal, 3(3) 692-713.

Cañal, P. y Porlán, R. (1987). Investigando la realidad próxima. Un módueo didáctico alternativo. Enseñanza de la Ciencias, 5(2), 89-96.

Cazden, C. (2001). Classroom discourse. The language of teaching and learning. USA: Heinemann.

Cicourel, A. (1974). Language Use and School Performance. Chicago: AcademicPress.

Chevallard, I. (1984). La trasposition didactique. Paris: La Pensée Sauvage.

Diamond, J. (1996). Status and power in verbal interaction. Amsterdam, Philadelphia, PA: John Benjamins.

Edwards, D. \& Potter, J. (1992). Discursive Psychology. London: Sage.

Erickson, F. (1982). Classroom discourse as improvisation: Relationship between Academic Task Structure and Social Participation in lessons. In L.C. Wilkinson (Comp.), Communicating in Classroom (pp. 153-181). New York: Academic Press.

Erickson, F. (2004). Talk and Social Theory: Ecologies of speaking and listening in everyday life. Cambridge: Cambridge University Press.

Jiménez-Aleixandre, M.P \& Puig, B. J. (2012). Argumentation, Evidence Evaluation and Critical Thinking. In B. Fraser, K. Tobin \& C. McRobbie (Eds), Second International Handbook of Science Education (Vol. 2, pp. 1001-1015). Dordrecht: Springer.

Latour, B. (1988). Science in action. Cambridge, MA: Harvard University Press.

Macias, J. (1992). The social nature of instruction in Mexican school. Implications for U.S. classroom practice. The Journal of Educational Issues of Language Monirity Students, 10, 13-25.

Mercer, N. (2001). Palabras y mentes: Cómo usamos el lenguaje para pensar juntos. Barcelona, Buenos Aires, México: Paidós.

Nespor, J. (1994). Knowledge in motion: Space, time and curriculum in undergraduate physics and management. London and New York: Routledge Falmer

Ravitck, D. (2010). The Death and Life of the Great American School System: How Testing and Choice are Undermining Education. New York: Basic Books.

Rockwell, E. (2009). La experiencia etnográfica: Historia y cultura en los procesos educativos. Buenos Aires: Paidós.

Paradise, R. \& Rogoff, B. (2009). Side by Side: Learning by Observing and Pitching In. Ethos: Journal of the Society for Psychological Anthropology, 39(1), 102-138.

https://doi.org/10.1111/j.1548-1352.2009.01033.x

Sinclair, J.M. \& Coulthard, M. (1975). Towards an analysis of discourse: The English used by teachers and pupils. London: Oxford University Press. 
Tobin, K. (2009). Tuning into others' voices: radical listening, learning from difference, and escaping oppression. Cultural Studies of Science Education, 4, 505- 511. DOI: 10.1007/s11422 009-9181-x

Topping, K. (2005). Trends in peer learning. Educational Psychology, 25(6), 631-641.

Vygotsky, L. (1984). Aprendizaje y desarrollo intelectual en la edad escolar. Infancia y Aprendizaje, 2728, 105-116.

Wells, G. (1993). Reevaluating the IRF sequence: A proposal for the articulation of theories of activity and discourse for the analysis of teaching and learning in the classroom. Linguistics and Education 5, 1-37.

Yager, R. E. \& Penich, J. E. (1983). Analysis of the current problems with school science in the USA. European Journal of Science Education, 5(4), 463-469.

\section{Información sobre la autora}

Autora: Antonia Candela

Institución: Centro de Investigación y de Estudios Avanzados del IPN

Email: acandela@cinvestav.mx

ORCID: https://orcid.org/0000-0002-9001-4451 


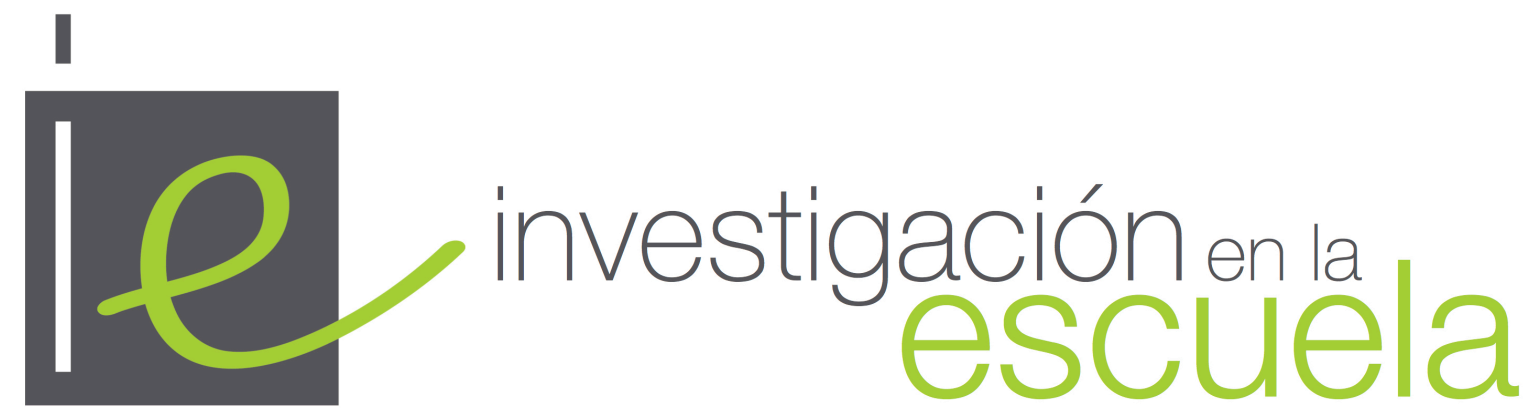

Revista académica evaluada por pares y de acceso abierto

Número 100

30 de abril de 2020

ISSN 2443-9991

\section{(c) (i) (2)}

Esta obra está bajo una licencia Creative Commons. Los/as lectores/as pueden compartir, copiar y redistribuir el material en cualquier medio o formato, así como adaptar, remezclar, transformar y construir a partir del material para cualquier propósito, incluso comercialmente. Para ello, deben de hacerlo bajo los siguientes términos: dando crédito de forma adecuada, brindando un enlace a la licencia e indicando si se han realizado cambios. Si se remezcla, transforma o crea a partir del material, debe distribuir su contribución bajo la misma licencia del original.

Más detalles de la licencia de CreativeCommons se encuentran en https://creativecommons.org/licenses/by-sa/4.0/deed.es

Cualquier otro uso debe ser aprobado en conjunto por el autor/es, o Investigación en la Escuela.

Uิ

Revista Editada por la Universidad de Sevilla. https://editorial.us.es/es/revistainvestigacion-en-la-escuela

Por errores y sugerencias contacte a investigacionenlaescuela@,us.es

La revista Investigación en la Escuela desde su origen en 1987 hasta su nº 87 (2015) fue editada por Díada Editora. 
